\title{
Assessment of Micronutrient Status of Hingoli and Sengaon Tahsils of Hingoli District, India
}

\author{
S.R. Adat*, T.R. Zagade and H.K. Kausadikar \\ Department of Soil Science and Agricultural Chemistry, Vasantrao Naik \\ Marathwada Krishi Vidyapeeth, Parbhani, India \\ *Corresponding author
}

\section{A B S T R A C T}

\begin{tabular}{|c|}
\hline Keywords \\
\hline $\begin{array}{l}\text { Soil fertility, } \\
\text { Micronutrient, } \\
\text { Fertilisers. }\end{array}$ \\
\hline Article Info \\
\hline $\begin{array}{l}\text { Accepted: } \\
12 \text { March } 2017 \\
\text { Available Online: } \\
10 \text { April } 2017\end{array}$ \\
\hline
\end{tabular}

\begin{abstract}
Although soil fertility mapping can help to apply appropriate fertilisers and effective fertility management techniques, there is still little information about spatial variability in fertility status of soil. Therefore this study was conducted at Hingoli and Sengaon tahsils with objects of assessing fertility status. From the study area 200 soil samples were collected and analysed in laboratory for micro nutrients. In micro nutrients Zinc indicate deficient condition followed by $\mathrm{Fe}, \mathrm{Cu}$ and $\mathrm{Mn}$ may be due to frequent cultivation, variations in management practices, high use of chemical fertilisers. DTPA- Zn content in soils of Hingoli tahsil were ranged from 0.14 to $1.75 \mathrm{mg} \mathrm{kg}^{-1}$ with a mean value of $0.57 \mathrm{mg}$ $\mathrm{kg}^{-1}$, DTPA- Fe content were ranged from 1.00 to $18.80 \mathrm{mg} \mathrm{kg}^{-1}$ with an average value of $5.92 \mathrm{mg} \mathrm{kg}^{-1}$, DTPA-Mn content varied from 0.88 to $19.70 \mathrm{mg} \mathrm{kg}^{-1}$ with a mean value of $6.58 \mathrm{mg} \mathrm{kg}^{-1}$. The available DTPA-Cu contents of Hingoli soils were ranged from 0.27 to $5.50 \mathrm{mg} \mathrm{kg}^{-1}$ with an average value of $1.73 \mathrm{mg} \mathrm{kg}^{-1}$. The data revealed from Sengaon tahsil that the available $\mathrm{Zn}$ content of these soils was ranged from 0.15 to $1.28 \mathrm{mg} \mathrm{kg}^{-1}$ with a mean value of $0.49 \mathrm{mg} \mathrm{kg}^{-1}$. The available Fe content of these soils was varied from 0.96 to $12.85 \mathrm{mg} \mathrm{kg}^{-1}$ with an average value of $5.90 \mathrm{mg} \mathrm{kg}^{-1}$. The available $\mathrm{Cu}$ content in the soils of Sengaon tahsil were ranged from 0.12 to $3.86 \mathrm{mg} \mathrm{kg}^{-1}$ with a mean value of 1.17 $\mathrm{mg} \mathrm{kg}^{-1}$. The lowest range 1.70 to $5.75 \mathrm{mg} \mathrm{kg}^{-1} \mathrm{Mn}$ with a mean value of $4.06 \mathrm{mg} \mathrm{kg}^{-1}$ were observed in Makodi whereas highest range 6.87 to $14.00 \mathrm{mg} \mathrm{kg}^{-1}$ with an average value of $9.72 \mathrm{mg} \mathrm{kg}^{-1} \mathrm{Mn}$ was recorded in village Wadhivra village. Therefore for increasing fertility of soil create public awareness about environmentally and socially acceptable integrated nutrient management practices like use of organic inputs, balanced use of chemical fertilisers, agro-forestry system and improved crop varieties can be adopted. Thus, it can be concluded that soils of Hingoli and Sengaon tahsil are low to medium in fertility status.
\end{abstract}

\section{Introduction}

Out of 329 million ha. area of the country, net cropped area 142 million ha. and about 175 million ha. suffers from different limitations. There is no additional land source available for horizontal expansion of soil to increase the net cultivated area, as the land to man ratio narrowing rapidly, the future requirement has to be met through intensification of agriculture (Sawashe, 2008).

Hingoli, a part of Parbhani district came into being as a district on may $1^{\text {st }} 1999$. Located in 
central Maharashtra but more nearer to Eastern district of Maharashtra with latitude and longitude of $19.43{ }^{0} \mathrm{~N}$ and $77.11{ }^{\circ} \mathrm{E}$ Hingoli district covered about 473400 ha geographical area with five tahsils Hingoli, Kalamnuri, Sengaon, Vasmath and Aundha Nagnath.

These Tahsils comes under Agro ecological unit No.6 except Kalamnuri which comes under Agro ecological unit No.8. About 421300 ha land is available for cultivation out of these total cultivable land, Kharif season shares 311200 and Rabi season 116400 ha.

The total geographical area of Hingoli is $4,73,400$ ha. and soils of this region varied in different physical and physico-chemical properties. Hingoli district comprises five tahsils and out of these two are selected for present study. Soil as well as crop differs significantly in their behavior due to their differential characteristics.

To know the present status of soil and future productivity, it is essential to know the fertility status. Considerable work on physicchemical properties of Maharastra soils was reported (Bharambe, 2001). Thus, it is necessary to define the areas of deficiency of particular nutrients in a particular areas and crops. Soil test data would be helpful in growing such deficient areas on soil and crop basis. Thus, the details of soil resource thematic maps and using data on various soil properties, focus given on fertility status, hence, present investigation is useful in judging the deficiencies of various nutrients.

\section{Materials and Methods}

Available $\mathrm{Cu}, \mathrm{Fe}, \mathrm{Mn}$ and $\mathrm{Zn}$ (DTPA extractable) were determined by using extraction method as described by Lindsay and Norvell (1978) using diethylene triamine penta acetic acid (DTPA), triethanolamine (TEA) extractant with the help of absorption spectroscopy by atomic absorption spectro meter model AA200 Perkin Elmer.

\section{Results and Discussion}

\section{Status of DTPA- Zn, Fe, Mn and $\mathrm{Cu}$ of Hingoli tahsil soils}

The data presented on DTPA-Zn and their categorization in revealed that, DTPA- Zn content in soils of Hingoli tahsil were ranged from 0.14 to $1.75 \mathrm{mg} \mathrm{kg}^{-1}$ with a mean value of $0.57 \mathrm{mg} \mathrm{kg}^{-1}$. The lowest $\mathrm{Zn}$ content was recorded in Ghota (sample no. HE2) while highest $\mathrm{Zn}$ content was observed in soils of Boralwadi village (sample no. HB3). The lowest range 0.14 to $0.89 \mathrm{mg} \mathrm{kg}^{-1}$ in $\mathrm{Zn}$ content with an average value of $0.34 \mathrm{mg} \mathrm{kg}^{-1}$ was recorded in Ghota where as highest range 0.22 to $1.75 \mathrm{mg} \mathrm{kg}^{-1}$ with a mean value of 0.82 were observed in Boralwadi village. Among twenty villages, 11 villages were low, while remaining, 9 were medium in $\mathrm{Zn}$ content. Out of 100 samples, 69 per cent in low $(<0.60 \mathrm{mg}$ $\left.\mathrm{kg}^{-1}\right), 21$ per cent in medium $(0.60$ to $1.20 \mathrm{mg}$ $\left.\mathrm{kg}^{-1}\right)$ and 10 per cent samples were categorized under high $\left(1.2 \mathrm{mg} \mathrm{kg} \mathrm{kg}^{-1}\right) \mathrm{Zn}$ content.

The values indicated that $\mathrm{Zn}$ content in Hingoli soils were low to medium. Majority of these soils were marginal in available $\mathrm{Zn}$ content. This might be due to DTPA extractable $\mathrm{Zn}$ decreases with depth because of low organic matter and variable in clay content. Also under alkaline condition the Zinc cations are charged largely to their oxides or hydroxides and thereby lower the availability of Zinc. Pharande et al., (1996) reported that Zinc status of Western Maharashtra ranged from 0.21 to $3.94 \mathrm{mg} \mathrm{kg}^{-1}$. The similar results were reported by Shinde, (2007).

Further, data presented in table indicated that the DTPA- Fe content of these soils were ranged from 1.00 to $18.80 \mathrm{mg} \mathrm{kg}^{-1}$ with an 
average value of $5.92 \mathrm{mg} \mathrm{kg}^{-1}$. The lowest Fe was recorded in (66sample no.HN1) and highest recorded in (sample no.HS4). The lowest range 1.34 to $3.88 \mathrm{mg} \mathrm{kg}^{-1}$ with a mean value of $2.49 \mathrm{mg} \mathrm{kg}^{-1}$ was recorded in village Ghota while highest range 3.62 to $18.80 \mathrm{mg}$ $\mathrm{kg}^{-1}$ in $\mathrm{Zn}$ content with an average value of $12.01 \mathrm{mg} \mathrm{kg}^{-1}$ was observed in soils of Pimpaldari village. Among twenty villages, 1 villages viz. Ghota $\left(2.49 \mathrm{mg} \mathrm{kg}^{-1}\right)$ was low in Fe contain, 8 villages were medium whereas 11 villages were high in $\mathrm{Fe}$ content (Tables 1 and 2).

Out of 100 samples, 17 per cent samples were low $\left(<2.5 \mathrm{mg} \mathrm{kg}^{-1}\right), 26$ per cent were medium ( 2.5 to $4.5 \mathrm{mg} \mathrm{kg}^{-1}$ ) and 47 per cent samples were high (> $4.5 \mathrm{mg} \mathrm{kg}^{-1}$ ) in DTPA-Fe content. These values showed that soils of Hingoli tahsils were low to high in available DTPA-Fe content. This high $\mathrm{Fe}$ content in soil may be due to presence of minerals like Feldspar, Magnetite, Haematite and Limonite which constitute bulk of trap rock in these soils. Malewar and Ismail (1999) reported that the available $\mathrm{Fe}$ content of Marathwada soils were ranges from 0.36 to $25.15 \mathrm{mg} \mathrm{kg}^{-1}$. These results were similar with the results reported by Pharande et al., (1996) and Jibhakate et al., (2009).

The available DTPA-Mn content of these soils was varied from 0.88 to $19.70 \mathrm{mg} \mathrm{kg}^{-1}$ with a mean value of $6.58 \mathrm{mg} \mathrm{kg}^{-1}$. The lowest value of Mn was recorded in village Pangari (sample no. HP4), while highest Mn content was observed in Digraj village (sample no.HK1). The lowest range 1.12 to $6.56 \mathrm{mg}$ $\mathrm{kg}^{-1}$ in Mn content with an average value of $3.51 \mathrm{mg} \mathrm{kg}^{-1}$ were observed in Pimpaldari whereas, highest range 6.95 to $15.16 \mathrm{mg} \mathrm{kg}^{-1}$ with a mean value of $10.51 \mathrm{mg} \mathrm{kg}^{-1}$ was recorded in Boralwadi village. Among twenty villages, 4 villages namely Hingoli $(4.21 \mathrm{mg}$ $\mathrm{kg}^{-1}$ ), Bhandegaon (4.22 $\mathrm{mg} \mathrm{kg}^{-1}$ ), Karanjali (3.97 $\mathrm{m} \mathrm{kg}^{-1}$ ) and Pimpaldari (3.51 $\mathrm{mg} \mathrm{kg}^{-1}$ ) were medium in $\mathrm{Mn}$ content whereas, remaining 16 villages were high in $\mathrm{Mn}$ content. Out of 100 sample, 16 per cent in low $\left(<2 \mathrm{mg} \mathrm{kg}^{-1}\right), 24$ per cent in medium (2 to 5 $\left.\mathrm{mg} \mathrm{kg}{ }^{-1}\right)$ and 60 per cent high $\left(>5 \mathrm{mg} \mathrm{kg}^{-1}\right)$ in Mn content. (Fig.7.3).

These value showed that soils of this tahsil were low to high in Mn content. The high status of Mn in these soils might due to the fact that lower Oxidation (Reduced) status of Mn are more soluble than higher oxidation state at Norman $\mathrm{pH}$ range of soil, Oxidation of divalent $\mathrm{Mn}^{++}$to trivalent $\mathrm{Mn}^{+++}$by certain Fungi and bacteria, also some organic compounds synthesized by micro-organisms or released by plans as root exudates have oxidizing or reducing power..The similar results were reported by Pharande et al., (1996) and Aage et al., (2007).

The available DTPA-Cu contents of Hingoli soils were ranged from 0.27 to $5.50 \mathrm{mg} \mathrm{kg}^{-1}$ with an average value of $1.73 \mathrm{mg} \mathrm{kg}^{-1}$. The lowest value $\left(0.27 \mathrm{mg} \mathrm{kg}^{-1}\right)$ of $\mathrm{Cu}$ content was recorded in village Pimpaldari, (sample no.HS2) where as highest value of $\mathrm{Cu}$ was recorded in soils of Takli village. (sample no.HT4). The lowest range 0.65 to $0.94 \mathrm{mg}$ $\mathrm{kg}^{-1}$ with mean value of $0.82 \mathrm{mg} \mathrm{kg}^{-1}$ were recorded in Takli village while highest range 0.56 to $5.50 \mathrm{mg} \mathrm{kg}^{-1}$ with an average value of $2.63 \mathrm{mg} \mathrm{kg}^{-1}$ were observed in Takli village in available $\mathrm{Cu}$ content in these soils of Hingoli tahsil. The soils of all selective villages were high in available $\mathrm{Cu}$ contents. Out of 100 samples, 3 per cent were low, 3 per cent were medium and 94 per cent high content of $\mathrm{Cu}$.

The high content of $\mathrm{Cu}$ in these soils were might be due to presence of $\mathrm{Cu}$ minerals like Cuprite and chalcocite, etc. in the parent material. Pharande et al., (1996) reported that the available status of $\mathrm{Cu}$ content of soil varied from 0.40 to $6.70 \mathrm{mg} \mathrm{kg}^{-1}$. Similar results were reported by Dhane et al., (1995), Dhage et al., (2000) and Malewar (1994). 
Table.1 Micronutrient status of Hingoli tahsil soils

\begin{tabular}{|c|c|c|c|c|c|}
\hline $\begin{array}{l}\text { Sr. } \\
\text { No. }\end{array}$ & $\begin{array}{c}\text { Sample } \\
\text { No. }\end{array}$ & $\begin{array}{c}\mathrm{Zn} \\
\left(\mathrm{mg} \mathrm{kg}^{-1}\right)\end{array}$ & $\begin{array}{c}\mathrm{Mn} \\
\left(\mathrm{mg} \mathrm{kg}^{-1}\right)\end{array}$ & $\begin{array}{c}\mathrm{Fe} \\
\left(\mathrm{mg} \mathrm{kg}^{-1}\right)\end{array}$ & $\begin{array}{c}\mathrm{Cu} \\
\left(\mathrm{mg} \mathrm{kg}^{-1}\right)\end{array}$ \\
\hline 1 & HA1 & 0.43 & 3.40 & 2.82 & 2.60 \\
\hline 2 & HA2 & 0.60 & 8.62 & 4.00 & 2.90 \\
\hline 3 & HA3 & 0.32 & 5.78 & 7.62 & 1.80 \\
\hline 4 & HA4 & 1.45 & 12.60 & 3.16 & 3.88 \\
\hline 5 & HA5 & 0.30 & 2.02 & 4.40 & 1.15 \\
\hline 6 & HB1 & 0.69 & 15.16 & 8.86 & 2.75 \\
\hline 7 & HB2 & 0.22 & 10.74 & 4.58 & 4.20 \\
\hline 8 & HB3 & 1.75 & 6.95 & 2.16 & 0.65 \\
\hline 9 & HB4 & 0.58 & 10.31 & 4.00 & 1.30 \\
\hline 10 & HB5 & 0.88 & 9.40 & 3.45 & 0.86 \\
\hline 11 & $\mathrm{HC} 1$ & 0.55 & 2.16 & 4.56 & 1.70 \\
\hline 12 & $\mathrm{HC} 2$ & 0.44 & 8.40 & 3.28 & 0.42 \\
\hline 13 & $\mathrm{HC} 3$ & 0.63 & 2.45 & 2.24 & 1.10 \\
\hline 14 & $\mathrm{HC} 4$ & 0.48 & 5.50 & 3.65 & 1.14 \\
\hline 15 & HC5 & 0.38 & 11.12 & 1.95 & 0.72 \\
\hline 16 & HD1 & 0.48 & 1.85 & 4.13 & 1.92 \\
\hline 17 & HD2 & 0.32 & 7.34 & 3.14 & 1.38 \\
\hline 18 & HD3 & 0.46 & 0.98 & 2.89 & 1.03 \\
\hline 19 & HD4 & 0.20 & 10.45 & 3.35 & 1.50 \\
\hline 20 & HD5 & 0.64 & 5.54 & 1.97 & 0.82 \\
\hline 21 & HE1 & 0.18 & 4.50 & 2.86 & 2.00 \\
\hline 22 & HE2 & 0.14 & 8.82 & 2.58 & 3.50 \\
\hline 23 & HE3 & 0.28 & 17.15 & 1.34 & 2.14 \\
\hline 24 & HE4 & 0.89 & 2.80 & 3.88 & 1.88 \\
\hline 25 & HE5 & 0.24 & 6.89 & 1.80 & 1.78 \\
\hline
\end{tabular}




\begin{tabular}{|c|c|c|c|c|c|}
\hline $\begin{array}{c}\text { Sr. } \\
\text { No. }\end{array}$ & $\begin{array}{c}\text { Sample } \\
\text { No. }\end{array}$ & $\begin{array}{c}\mathbf{Z n} \\
\left(\mathbf{m g ~ k g}^{-1}\right)\end{array}$ & $\begin{array}{c}\mathbf{M n} \\
\left(\mathbf{m g ~ k g}^{-1}\right)\end{array}$ & $\begin{array}{c}\mathbf{F e} \\
\left(\mathbf{m g ~ k g}^{-1}\right)\end{array}$ & $\begin{array}{c}\mathbf{C u} \\
\left(\mathbf{m g ~ k g}^{-1}\right)\end{array}$ \\
\hline 26 & HF1 & 0.54 & 1.78 & 2.63 & 2.16 \\
\hline 27 & HF2 & 0.36 & 9.72 & 1.46 & 0.82 \\
\hline 28 & HF3 & 1.22 & 3.78 & 2.44 & 0.92 \\
\hline 29 & HF4 & 0.55 & 12.40 & 4.95 & 0.84 \\
\hline 30 & HF5 & 0.35 & 4.90 & 6.20 & 0.98 \\
\hline 31 & HG1 & 1.04 & 1.72 & 1.88 & 4.42 \\
\hline 32 & HG2 & 0.20 & 2.42 & 5.78 & 2.14 \\
\hline 33 & HG3 & 0.30 & 9.22 & 5.26 & 3.50 \\
\hline 34 & HG4 & 0.36 & 10.48 & 5.00 & 2.58 \\
\hline 35 & HG5 & 0.24 & 9.18 & 7.20 & 0.91 \\
\hline 36 & HH1 & 0.42 & 4.20 & 7.10 & 0.85 \\
\hline 37 & HH2 & 0.32 & 1.23 & 6.34 & 0.75 \\
\hline 38 & HH3 & 0.45 & 7.84 & 8.32 & 0.94 \\
\hline 39 & HH4 & 0.58 & 6.22 & 5.34 & 0.65 \\
\hline 40 & HH5 & 0.94 & 1.58 & 5.92 & 0.91 \\
\hline 41 & HI1 & 1.20 & 5.28 & 4.22 & 1.05 \\
\hline 42 & HI2 & 0.34 & 2.02 & 5.46 & 2.46 \\
\hline 43 & HI3 & 1.28 & 14.16 & 5.17 & 1.34 \\
\hline 44 & HI4 & 0.42 & 4.20 & 1.80 & 0.32 \\
\hline 45 & HI5 & 0.58 & 9.06 & 4.42 & 0.88 \\
\hline 46 & HJ1 & 0.43 & 0.98 & 6.34 & 0.72 \\
\hline 47 & HJ2 & 0.81 & 2.26 & 5.42 & 1.80 \\
\hline 48 & HJ3 & 0.44 & 11.3 & 7.21 & 2.44 \\
\hline 49 & HJ4 & 0.68 & 6.96 & 2.47 & 1.32 \\
\hline 50 & HJ5 & 1.44 & 3.64 & 3.15 & 1.72 \\
\hline
\end{tabular}




\begin{tabular}{|c|c|c|c|c|c|}
\hline $\begin{array}{c}\text { Sr. } \\
\text { No. }\end{array}$ & $\begin{array}{c}\text { Sample } \\
\text { No. }\end{array}$ & $\begin{array}{c}\mathbf{Z n} \\
\left(\mathbf{m g ~ k g} \mathbf{~}^{-1}\right)\end{array}$ & $\begin{array}{c}\mathbf{M n} \\
\left(\mathbf{m g ~ k g}^{-\mathbf{1}}\right)\end{array}$ & $\begin{array}{c}\mathbf{F e} \\
\left(\mathbf{m g ~ k g}^{-\mathbf{1}}\right)\end{array}$ & $\begin{array}{c}\mathbf{C u} \\
\left(\mathbf{m g ~ k g}^{-\mathbf{1}}\right)\end{array}$ \\
\hline 51 & HK1 & 0.84 & 19.70 & 6.09 & 2.40 \\
\hline 52 & HK2 & 0.28 & 5.50 & 11.16 & 0.91 \\
\hline 53 & HK3 & 0.89 & 1.68 & 9.48 & 0.38 \\
\hline 54 & HK4 & 0.82 & 6.50 & 5.18 & 0.71 \\
\hline 55 & HK5 & 0.54 & 8.44 & 10.04 & 2.92 \\
\hline 56 & HL1 & 0.62 & 1.90 & 11.40 & 1.49 \\
\hline 57 & HL2 & 0.50 & 3.15 & 8.10 & 3.78 \\
\hline 58 & HL3 & 0.57 & 14.20 & 2.30 & 4.90 \\
\hline 59 & HL4 & 0.44 & 11.62 & 12.48 & 0.28 \\
\hline 60 & HL5 & 0.51 & 6.41 & 12.85 & 0.82 \\
\hline 61 & HM1 & 0.67 & 3.45 & 12.48 & 2.42 \\
\hline 62 & HM2 & 0.84 & 8.42 & 9.78 & 2.82 \\
\hline 63 & HM3 & 0.45 & 2.47 & 10.65 & 0.94 \\
\hline 64 & HM4 & 0.18 & 11.10 & 6.08 & 3.50 \\
\hline 65 & HM5 & 0.42 & 1.42 & 3.64 & 1.88 \\
\hline 66 & HN1 & 0.18 & 7.32 & 1.00 & 4.42 \\
\hline 67 & HN2 & 0.72 & 9.48 & 6.40 & 0.70 \\
\hline 68 & HN3 & 1.40 & 5.64 & 9.80 & 1.08 \\
\hline 69 & HN4 & 0.34 & 1.32 & 14.22 & 0.68 \\
\hline 70 & HN5 & 0.46 & 10.88 & 11.48 & 0.34 \\
\hline 71 & HO1 & 0.43 & 10.80 & 4.20 & 1.72 \\
\hline 72 & HO2 & 0.49 & 4.94 & 8.82 & 0.84 \\
\hline 73 & HO3 & 0.54 & 2.04 & 4.58 & 1.31 \\
\hline 74 & HO4 & 1.27 & 1.22 & 2.16 & 2.70 \\
\hline 75 & HO5 & 0.78 & 2.10 & 2.24 & 1.22 \\
\hline
\end{tabular}




\begin{tabular}{|c|c|c|c|c|c|}
\hline $\begin{array}{l}\text { Sr. } \\
\text { No. }\end{array}$ & $\begin{array}{c}\text { Sample } \\
\text { No. }\end{array}$ & $\begin{array}{c}\mathrm{Zn} \\
\left(\mathrm{mg} \mathrm{kg}^{-1}\right)\end{array}$ & 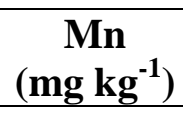 & $\begin{array}{c}\mathrm{Fe} \\
\left(\mathrm{mg} \mathrm{kg}^{-1}\right)\end{array}$ & $\underset{\left(\mathrm{mg} \mathrm{kg}^{-1}\right)}{\mathrm{Cu}}$ \\
\hline 76 & HP1 & 0.40 & 5.78 & 3.14 & 0.68 \\
\hline 77 & HP2 & 0.68 & 4.30 & 1.86 & 0.92 \\
\hline 78 & HP3 & 0.54 & 8.42 & 2.65 & 0.74 \\
\hline 79 & HP4 & 0.32 & 0.88 & 6.78 & 1.19 \\
\hline 80 & HP5 & 0.44 & 11.62 & 7.20 & 2.32 \\
\hline 81 & HQ1 & 1.22 & 10.40 & 10.32 & 2.42 \\
\hline 82 & HQ2 & 0.72 & 6.22 & 5.18 & 3.52 \\
\hline 83 & HQ3 & 0.58 & 19.20 & 11.5 & 3.62 \\
\hline 84 & HQ4 & 0.30 & 6.49 & 5.18 & 0.85 \\
\hline 85 & HQ5 & 0.26 & 7.32 & 12.86 & 2.12 \\
\hline 86 & HR1 & 0.18 & 11.38 & 8.10 & 2.32 \\
\hline 87 & HR2 & 0.31 & 2.70 & 9.42 & 2.00 \\
\hline 88 & HR3 & 0.72 & 5.20 & 7.78 & 1.66 \\
\hline 89 & HR4 & 0.41 & 1.84 & 12.04 & 2.14 \\
\hline 90 & HR5 & 0.82 & 8.76 & 4.82 & 1.50 \\
\hline 91 & HS1 & 0.44 & 1.97 & 3.62 & 0.72 \\
\hline 92 & HS2 & 0.34 & 4.10 & 18.36 & 0.27 \\
\hline 93 & HS3 & 0.25 & 6.56 & 9.66 & 0.81 \\
\hline 94 & HS4 & 0.38 & 3.84 & 18.8 & 1.12 \\
\hline 95 & HS5 & 0.48 & 1.12 & 9.65 & 1.56 \\
\hline 96 & HT1 & 0.54 & 10.10 & 1.00 & 3.10 \\
\hline 97 & HT2 & 0.57 & 8.82 & 3.45 & 2.80 \\
\hline 98 & HT3 & 1.29 & 5.52 & 4.94 & 0.56 \\
\hline 99 & HT4 & 0.35 & 15.48 & 3.20 & 5.50 \\
\hline \multirow[t]{2}{*}{100} & HT5 & 0.38 & 1.69 & 3.68 & 1.20 \\
\hline & Mean & 0.57 & 6.58 & 5.92 & 1.73 \\
\hline
\end{tabular}


Int.J.Curr.Microbiol.App.Sci (2017) 6(4): 1441-1452

Table.2 Micronutrients status of Sengaon tahsil

\begin{tabular}{|c|c|c|c|c|c|}
\hline Sr.No. & $\begin{array}{c}\text { Sample } \\
\text { No. }\end{array}$ & $\begin{array}{c}\text { Fe } \\
\left(\mathrm{mg} \mathrm{kg}^{-1}\right)\end{array}$ & $\begin{array}{c}\mathrm{Mn} \\
\left(\mathrm{mg} \mathrm{kg}^{-1}\right)\end{array}$ & $\begin{array}{c}\mathrm{Zn} \\
\left(\mathrm{mg} \mathrm{kg}^{-1}\right)\end{array}$ & $\begin{array}{c}\mathrm{Cu} \\
\left(\mathrm{mg} \mathrm{kg}^{-1}\right)\end{array}$ \\
\hline 1 & SA1 & 3.45 & 11.38 & 0.81 & 2.32 \\
\hline 2 & SA2 & 1.42 & 8.72 & 0.36 & 0.68 \\
\hline 3 & SA3 & 3.12 & 2 & 0.42 & 2 \\
\hline 4 & SA4 & 6.2 & 10.44 & 0.23 & 2.16 \\
\hline 5 & SA5 & 4.96 & 14.92 & 0.38 & 0.83 \\
\hline 6 & SB1 & 1.08 & 2.34 & 1.22 & 1.18 \\
\hline 7 & SB2 & 5.3 & 6.56 & 0.19 & 0.32 \\
\hline 8 & SB3 & 4.38 & 6.85 & 0.88 & 0.28 \\
\hline 9 & SB4 & 5.31 & 3.12 & 0.38 & 0.64 \\
\hline 10 & SB5 & 8.97 & 1.7 & 0.48 & 1.32 \\
\hline 11 & SC1 & 5.42 & 10.43 & 1.28 & 2.78 \\
\hline 12 & $\mathrm{SC} 2$ & 8.98 & 8.45 & 0.42 & 2.34 \\
\hline 13 & SC3 & 7.21 & 2.78 & 0.54 & 2 \\
\hline 14 & SC4 & 2.54 & 8.76 & 0.52 & 3.18 \\
\hline 15 & SC5 & 6.34 & 5.5 & 0.25 & 0.62 \\
\hline 16 & SD1 & 5.44 & 1.05 & 1.21 & 0.74 \\
\hline 17 & SD2 & 4.42 & 6.3 & 0.91 & 0.98 \\
\hline 18 & SD3 & 8.48 & 2.22 & 0.33 & 1.05 \\
\hline 19 & SD4 & 7.52 & 5.78 & 0.21 & 1.77 \\
\hline 20 & SD5 & 8.9 & 9.6 & 0.54 & 0.98 \\
\hline 21 & SE1 & 5.1 & 6.65 & 0.77 & 0.12 \\
\hline 22 & SE2 & 4.42 & 1.7 & 0.55 & 0.94 \\
\hline 23 & SE3 & 3.36 & 7.78 & 0.21 & 0.58 \\
\hline 24 & SE4 & 2.05 & 3.2 & 1.28 & 0.75 \\
\hline 25 & SE5 & 5.18 & 10.4 & 0.15 & 0.98 \\
\hline 26 & SF1 & 10.32 & 8.45 & 0.28 & 1.3 \\
\hline 27 & SF2 & 11.22 & 2.84 & 0.18 & 1.72 \\
\hline 28 & SF3 & 8.48 & 6.68 & 0.72 & 1.24 \\
\hline 29 & SF4 & 1.46 & 9.72 & 0.45 & 1.14 \\
\hline 30 & SF5 & 6.5 & 6.6 & 0.38 & 2.32 \\
\hline 31 & SG1 & 7.22 & 10.28 & 0.27 & 2.6 \\
\hline 32 & SG2 & 1.8 & 8.42 & 0.43 & 0.96 \\
\hline 33 & SG3 & 4.8 & 4.2 & 0.32 & 0.26 \\
\hline 34 & SG4 & 2.98 & 8.72 & 0.54 & 2.2 \\
\hline 35 & SG5 & 6.2 & 6.9 & 1.26 & 0.5 \\
\hline 36 & SH1 & 7.35 & 5 & 0.5 & 1.7 \\
\hline 37 & $\mathrm{SH} 2$ & 3.88 & 3.12 & 0.62 & 0.95 \\
\hline 38 & SH3 & 1.94 & 7.32 & 0.79 & 0.38 \\
\hline 39 & SH4 & 6.34 & 11.3 & 0.4 & 1.22 \\
\hline 40 & SH5 & 7.81 & 1.26 & 0.42 & 1.5 \\
\hline 41 & SI1 & 4.26 & 5.56 & 0.3 & 2.68 \\
\hline
\end{tabular}




\begin{tabular}{|c|c|c|c|c|c|}
\hline 42 & SI2 & 8.92 & 12.28 & 0.38 & 0.98 \\
\hline 43 & SI3 & 0.98 & 7.1 & 0.26 & 1.04 \\
\hline 44 & SI4 & 4.55 & 6.05 & 0.18 & 2.14 \\
\hline 45 & SI5 & 7.25 & 4.78 & 0.22 & 0.78 \\
\hline 46 & SJ1 & 8.44 & 3.04 & 0.3 & 1.25 \\
\hline 47 & SJ2 & 3.22 & 5.75 & 0.54 & 1.74 \\
\hline 48 & $\begin{array}{l}\text { SJ3 } \\
\end{array}$ & 5.48 & 1.7 & 0.44 & 0.42 \\
\hline 49 & SJ4 & 7.5 & 5.5 & 0.28 & 1.56 \\
\hline 50 & SJ5 & 12.5 & 4.34 & 1.2 & 0.75 \\
\hline 51 & SK1 & 3.45 & 2.02 & 0.62 & 1.72 \\
\hline 52 & SK2 & 6.9 & 11.3 & 1 & 0.21 \\
\hline 53 & SK3 & 1.52 & 6.22 & 0.41 & 0.86 \\
\hline 54 & SK4 & 9.88 & 7.78 & 0.58 & 1.42 \\
\hline 55 & SK5 & 10.34 & 0.98 & 0.56 & 0.96 \\
\hline 56 & SL1 & 2.92 & 7.55 & 0.2 & 2.9 \\
\hline 57 & SL2 & 7.08 & 5.91 & 0.32 & 0.48 \\
\hline 58 & SL3 & 2.35 & 12.24 & 0.36 & 0.72 \\
\hline 59 & SL4 & 5.44 & 12.74 & 0.2 & 1.02 \\
\hline 60 & SL5 & 3.22 & 9.34 & 0.43 & 0.62 \\
\hline 61 & SM1 & 3.12 & 11.37 & 0.28 & 0.97 \\
\hline 62 & SM2 & 4.5 & 2.7 & 0.42 & 1.92 \\
\hline 63 & SM3 & 8.36 & 2 & 0.68 & 2.84 \\
\hline 64 & SM4 & 9.48 & 5.2 & 0.32 & 0.71 \\
\hline 65 & SM5 & 1.2 & 8.72 & 0.22 & 0.22 \\
\hline 66 & SN1 & 6.35 & 14.32 & 1.23 & 0.99 \\
\hline 67 & SN2 & 10.04 & 6.56 & 0.26 & 1.21 \\
\hline 68 & SN3 & 9.6 & 2.76 & 0.81 & 1.08 \\
\hline 69 & SN4 & 5.18 & 12.32 & 0.36 & 0.6 \\
\hline 70 & SN5 & 11.4 & 15.14 & 0.18 & 0.68 \\
\hline 71 & SO1 & 2.28 & 6.87 & 0.15 & 0.88 \\
\hline 72 & $\mathrm{SO} 2$ & 12.85 & 8.34 & 0.35 & 1.5 \\
\hline 73 & SO3 & 7.1 & 14 & 0.38 & 0.48 \\
\hline 74 & SO4 & 5.42 & 8.8 & 0.4 & 0.72 \\
\hline 75 & SO5 & 9.12 & 10.7 & 0.25 & 0.78 \\
\hline 76 & SP1 & 8.1 & 9.82 & 0.51 & 0.72 \\
\hline 77 & SP2 & 0.96 & 2.75 & 0.63 & 2.12 \\
\hline 78 & SP3 & 2.68 & 9.7 & 0.17 & 0.79 \\
\hline 79 & SP4 & 6.48 & 1.3 & 0.55 & 1.12 \\
\hline 80 & SP5 & 9.35 & 6.45 & 1.28 & 0.3 \\
\hline 81 & SQ1 & 7.45 & 12.2 & 0.33 & 1.26 \\
\hline 82 & SQ2 & 8.87 & 11.18 & 0.19 & 1.3 \\
\hline 83 & SQ3 & 2.15 & 9.1 & 0.41 & 1.9 \\
\hline 84 & SQ4 & 12.5 & 6.71 & 0.34 & 0.62 \\
\hline 85 & SQ5 & 4.72 & 5.98 & 1.22 & 0.78 \\
\hline 86 & SR1 & 3.75 & 6.45 & 0.37 & 0.32 \\
\hline
\end{tabular}




\begin{tabular}{|c|c|c|c|c|c|}
\hline 87 & SR2 & 12.6 & 8.36 & 0.24 & 1.12 \\
\hline 88 & SR3 & 5.3 & 10.6 & 0.32 & 2.2 \\
\hline 89 & SR4 & 4.28 & 6.9 & 0.25 & 0.98 \\
\hline 90 & SR5 & 8.68 & 8.72 & 0.2 & 0.65 \\
\hline 91 & SS1 & 8.3 & 7.35 & 0.47 & 2 \\
\hline 92 & SS2 & 1 & 5.2 & 1.21 & 0.95 \\
\hline 93 & SS3 & 5.92 & 2.31 & 0.19 & 0.2 \\
\hline 94 & SS4 & 2.44 & 8.4 & 0.38 & 1.36 \\
\hline 95 & SS5 & 3.89 & 10.1 & 0.49 & 0.71 \\
\hline 96 & ST1 & 10.65 & 12.45 & 0.71 & 2.05 \\
\hline 97 & ST2 & 5.13 & 11.6 & 1.25 & 0.37 \\
\hline 98 & ST3 & 4.42 & 6.95 & 0.45 & 0.91 \\
\hline 99 & ST4 & 7.22 & 9.85 & 0.23 & 0.67 \\
\hline 100 & ST5 & 8.32 & 7.7 & 0.35 & 0.75 \\
\hline & Mean & $\mathbf{5 . 9 5}$ & $\mathbf{7 . 1 9}$ & $\mathbf{0 . 4 9}$ & $\mathbf{1 . 1 7}$ \\
\hline
\end{tabular}

Status of available $\mathrm{Zn}, \mathrm{Fe}$, Mn and $\mathrm{Cu}$ of Sengaon tahsil of soils

The data revealed that the available $\mathrm{Zn}$ content of these soils were ranged from 0.15 to $1.28 \mathrm{mg}$ $\mathrm{kg}^{-1}$ with a mean value of $0.49 \mathrm{mg} \mathrm{kg}^{-1}$. The lowest $\mathrm{Zn}$ content was observed in village Wadhivra (sample no. SO1) while highest $\mathrm{Zn}$ content was recorded in Bhankheda, Goregaon and Sengi village (sample no.SC2, SE4 and SP5). The lowest range 0.18 to $0.38 \mathrm{mg} \mathrm{kg}^{-1}$ with a mean value of $0.26 \mathrm{mg} \mathrm{kg}^{-1}$ were observed in village Kendre where as highest range 0.21 to $1.21 \mathrm{mg} \mathrm{kg}^{-1}$ with an average value of $0.64 \mathrm{mg} \mathrm{kg}^{-1}$ were recorded in Chaundi village. Out of Twenty villages, 17 villages were low in $\mathrm{Zn}$ content whereas, remaining villages viz., Ajegaon $\left(0.63 \mathrm{mg} \mathrm{kg}{ }^{-1}\right)$,Mhalsi (0.0.63 mg kg $\mathrm{mg}^{-1}$ ) and Sengi (0.62) villages were moderate in $\mathrm{Zn}$ content. Among 100 soil samples, 76 per cent low, 14 per cent moderate and 10 per cent were categorized in high $\mathrm{Zn}$ content.

The above values indicated that the soils of Sengaon tahsil were low to high in $\mathrm{Zn}$ content. The most of the soils were low in $\mathrm{Zn}$ content. In well drained aerated calcareous soils Zinc exist in oxidized state and its availability become low. The similar results were also reported by Pharande et al., (1996) and Waghmare (2007).
The available Fe content of these soils were varied from 0.96 to $12.85 \mathrm{mg} \mathrm{kg}^{-1}$ with an average value of $5.90 \mathrm{mg} \mathrm{kg}{ }^{-1}$. The low $\mathrm{Fe}$ content was recorded in village Sengi (sample no. SP2) while high Fe content was observed in village Wadhivra (sample no. SO2). The lowest range 1.42 to $6.20 \mathrm{mg} \mathrm{kg}^{-1}$ with a mean value of $3.83 \mathrm{mg} \mathrm{kg}^{-1}$ in $\mathrm{Fe}$ content were recorded in Adul village while highest range 1.46 to 11.22 $\mathrm{mg} \mathrm{kg}^{-1}$ with an average value of $7.59 \mathrm{mg} \mathrm{kg}^{-1}$ Fe content were observed in village Gondala. Villages of Sengaon tahsil were high in $\mathrm{Fe}$ content. Out of 20 villages, 4 villages were medium and 16 villages were high in $\mathrm{Fe}$ content. Out of 100 samples, 13 per cent samples in low, 20 per cent were medium and 67 per cent were categorized in high Fe content.

Maximum soils of these tahsil were rich in $\mathrm{Fe}$ content. This could be attributed to silicate clays contains Fe in octahedral layer especially in 2: 1 type of clay minerals (Smectite). Fe released from the clay under certain soil conditions on their concentrations in soil solution is increased by fertilizer application of Fe content. Malewar and Ismail (1999) reported that the soils of Marathwada region were ranges from 0.36 to $25.14 \mathrm{mg} \mathrm{kg}$. These results were in confirmatory with result reported by Meena et al., (2006) and Shinde (2007). 
Further data revealed that the available $\mathrm{Mn}$ content in these soils was varied from 0.98 to $15.14 \mathrm{mg} \mathrm{kg}^{-1}$ with a mean value of $7.19 \mathrm{mg} \mathrm{kg}$ 1. The lowest $\mathrm{Mn}$ content $\left(0.98 \mathrm{mg} \mathrm{kg}^{-1}\right)$ was recorded in soils of Mhalshi village (sample no.SK5) while highest $\mathrm{Mn}$ content (15.14 mg $\mathrm{kg}^{-1}$ ) was found in soils of Sapatgaon village.(sample no.SN5).The lowest range 1.70 to $5.75 \mathrm{mg} \mathrm{kg}^{-1} \mathrm{Mn}$ with a mean value of 4.06 mg $\mathrm{kg}^{-1}$ were observed in Makodi whereas highest range 6.87 to $14.00 \mathrm{mg} \mathrm{kg}^{-1}$ with an average value of $9.72 \mathrm{mg} \mathrm{kg}^{-1} \mathrm{Mn}$ was recorded in village Wadhivra. Out 20 villages, 3 villages Viz. Ajegaon (4.11 $\mathrm{mg} \mathrm{kg}^{-1}$ ), Chundi $(4.99 \mathrm{mg}$ $\mathrm{kg}^{-1}$ ) and Makodi (4.06 $\mathrm{mg} \mathrm{kg} \mathrm{kg}^{-1}$ ) were categorized medium and remaining 17 villages were categorized in high content of $\mathrm{Mn}$. Among the 100 soil samples, 15 percent in moderate and 85 percent samples were high in $\mathrm{Mn}$ content.

The relative high content of $\mathrm{Mn}$ in these soils could be due to the soils derived from basaltic parent material which contained higher ferromagnessiume minerals. Hundal et al., (2006) reported that the available Mn content were ranged from 0.07 to $18.56 \mathrm{mg} \mathrm{kg}^{-1}$. Similar findings were reported by Malewar and Ismail (1999).

The available $\mathrm{Cu}$ content in the soils of Sengaon tahsil were ranged from 0.12 to $3.86 \mathrm{mg} \mathrm{kg}^{-1}$ with a mean value of $1.17 \mathrm{mg} \mathrm{kg}^{-1}$. The lowest $\mathrm{Cu}$ content was observed in soils of Goregaon village (sample no.SE1) whereas, highest $\mathrm{Cu}$ content was found in soils of Mhalshi village. (sample no. SK3). The lowest range 0.12 to $0.98 \mathrm{mg} \mathrm{kg}^{-1}$ with an average value of $0.67 \mathrm{mg}$ $\mathrm{kg}^{-1}$ in $\mathrm{Cu}$ content was observed in village Goregaon whereas, highest range 0.62 to 3.18 $\mathrm{mg} \mathrm{kg}^{-1}$ with a mean value of $2.18 \mathrm{mg} \mathrm{kg}^{-1}$ was recorded in Bhankheda village. The villages of Sengaon tahsil (100 samples) were categorized as, 7 per cent under low, 8 per cent under medium and 85 per cent high $\mathrm{Cu}$ content.The high content of $\mathrm{Cu}$ in these soils could be attributed to the difference in geology, physiology and degree of weathering in these soils. Malewar (1994) reported that the $\mathrm{Cu}$ content in Marathwada soil were varied from 1.2 to $7.40 \mathrm{mg} \mathrm{kg}^{-1}$. Similar results also reported by Dhage et al., (2000) and Gupta et al., (2003).

\section{References}

Aage, A.B., Magar, S.M., Godhawale, G.V. and Borgaonkar, S.B. 2007a. Studies on Available micronutrient status in Beed district of Maharashtra state. Int. J. Trop. Agri., 25(3): 491-494.

Basavaraju, N.G., Mir Khamir Ali and Ravi, M.V. 2008. Distribution of zinc and their relationship with physico- chemical properties in Black soils of Sira Taluka Tumkur district, Karnataka. Maysore J. Agric. Sci., 42(3): 450-455.

Bharmbe, P.R. and Ghonshikar, C.P. 1984. Fertility status of soils in Jayakwadi command. J. Maharastra Agric. Univ., 9(3): 326-327.

Binita, N.K., Dasog, G.S. and Patil, P.L. 2009. Soil fertility mapping in Ghatprabha left bank canal command area of north Karnataka by geographic information system technique. Karnataka J. Agric. Sci., 22(1): 81-88.

Black, C.A. 1965. Methods of soil Analysis part-2. American society of Agron. In. C. Madison Wiscousin, USA.

Chopra, S.L. and Kanwar, J.B. 1976. Analytical Agricultural Chemistry. Kalyani publ., New Delhi.

Dhage, A.R., Mane, S.R. and Adsule, R.N. 2000. Available micronutrient in soils of Shevgaon tahsil (Ahmadnagar) in relation to soil characteristics. J. Maharashtra Agric. Univ., 35(1): 97-98.

Dhane, S.S. and Shukla, L.M. 1995. Distribution of DTPA extractable $\mathrm{Zn}, \mathrm{Cu}$, $\mathrm{Mn}$ and $\mathrm{Fe}$ in some soil series of Maharashtra and their relationship with some soil properties. J. Indian Soc. Soil., 43(4): 597-600.

Dhanya, V., Mathews., Patil, P.L. and Dasog, G.S. 2009. Identification of soil fertility constraints of a pilot site in coastal agro ecosystem of Karnataka by GIS 
techniques. Karnataka J. Agric. Sci., 22(1): 77-80.

Dolui, A.K. and R. Bera. 2001. Soil tests for available Iron and their Relation with soil properties in some Alfisols of Orissa India. Int. J. Trop. Agri., 19(1-4): 5-15.

Dwivedi, S.K., Sharma, V.K. and Bhardwaj, Vipin. 2005. Status of available nutrients in soils of cold arid region of Ladakh. $J$. Indian Soc. Soil Sci., 53(3): 421-423.

Gupta Naresh, S.K., Trivedi, K.N., Bansal and Kaul, R.K. 2003. Vertical distribution of micronutrient cations in some soil series of Northern Madhya Pradesh. J. Indian Soc. Soil. Sci., 51(4): 517-522.

Hegde, D.M. and Sudhakara Bapu, S.N. 2001. Nutrient management strategies in Agriculture, A future outlook. Fertilizer News, 46(12): 61-66.

Jibhkate, S.B., Raut, M.M., Bhende, S.N. and Kharche, V.K. 2009. Micronutrient status of soils of Katol tahsil in Nagpur district and their Relationship with some soil properties. J. Soils and Crops, 19(1): 143146.

Krishna Murthy and Srinivas Murthy. 2005. Distribution of some available micronutrients in black and red soils of Karnataka. Mysore J. Agric. Sci., 39(1): 57-63.

Lindsay, W.L. and Norvell, W.A. 1978. Development of DTPA soil test for Zn, $\mathrm{Fe}, \mathrm{Mn}$ and $\mathrm{Cu}$. Soil Sci. Soc. Amer. J., 42: 421-425.

Mahapatra, P.K. and Sahu, S.K. 1996. Relationship between some major, secondary and micronutrient status of soils growing groundnut and their contents in groundnut plants. J. Indian Soc. Soil. Sci., 44(1): 100-103.

Malewar, G.U., Dhamak, A.L. and Ismail, S. 1998. Inter relationship of forms of $\mathrm{Fe}$ with properties of soils of semi arid area of Northen Marathwada J. Maharashtra Agric. Univ., 23(2): 201-202.

Malewar, G.U. and Syed Ismail. 1999. Increasing significance of micronutrients in sugarcane production special paper presented at $\mathrm{XV}^{\text {th }}$ cane development workshop on soil fertility Management Vasantdada Sugar Institute, Manjari (Pune) Sept. 17-18, 1999 PP : 24-32.

Malewar, G.U. and Ghonshikar, C.P. 1984. Maharashtra soils and their Zinc chemistry. J. Maharashtra Agric. Univ., 9(1): 1-7.

Mali, C.V. and Syed Ismail. 2002. Characterization and fertility status of Marathwada soils sulphur and micronutrient in soil resource inventory of Marathwada Departments of Agricultural chemistry and soil science. MAU Parbhani,72-57.

Mali, C.V., Gorde, A.S., Rekhawar, B.V. and Malewar, G.U. 1994. Micronutient status of some sweet orange growing soils in Aurangabad district. Paper published in seminar on recent trends in Micronutrient Research in soils and plants in Maharashtra, organized by PCISSS, at Marathwada Agril. Univ. Nov. 12 \& 13, 1994 Abst., PP. 18.

\section{How to cite this article:}

Adat, S.R., T.R. Zagade and Kausadikar, H.K. 2017. Assessment of Micronutrient Status of Hingoli and Sengaon Tahsils of Hingoli District. Int.J.Curr.Microbiol.App.Sci. 6(4): 1441-1452.

doi: https://doi.org/10.20546/ijcmas.2017.604.176 